\title{
Abundance Gradients and Chemical Evolution of Spiral Galaxies
}

\author{
Monica M. Marcon-Uchida ${ }^{1,2}$, Francesca Matteucci ${ }^{1,3}$ \\ and Roberto D. D. Costa ${ }^{2}$ \\ ${ }^{1}$ Dipartamento di Astronomia, Università degli Studi di Trieste, \\ Via G.B. Tiepolo 11, I-34131, Trieste, Italy \\ ${ }^{2}$ Instituto de Astronomia, Geofísica e Ciéncias Atmosféricas(IAG), Universidade de São Paulo, \\ Rua do Matão, 1226 05508-090 São Paulo, Brazil \\ ${ }^{3}$ I.N.A.F Osservatorio Astronomico di Trieste, \\ Via G.B. Tiepolo 11, I-34131, Trieste, Italy \\ email: monica@astro.iag.usp.br, roberto@astro.iag.usp.br, matteucc@oats.inaf.it
}

\begin{abstract}
The distribution of chemical abundances and their variation in space and time are important tools to understand the chemical evolution of disks in spiral galaxies. In this work we present an one infall chemical evolution model for the Galactic disk based on an updated version of the Trieste group model. We adopted a pre enriched gas (to take into account the effect of the halo evolution), an inside-out scenario for the formation of the disk and a threshold in the surface gas density to regulate the star formation rate. The observational constraints for the solar neighbourhood were well reproduced and the spatial and time evolution of the radial abundance gradient were studied. We also used this model to reproduce the chemical evolution of some nearby spiral galaxies. The model was scaled to the disk properties of each galaxy and its dependence with the star formation efficiency and the time scale for the infalling gas into the disk were explored. Using this modified model we were able to reproduce the observed constraints available in the literature for this galaxies. The similarities and the differences between the chemical evolution of these objects and teh Milky Way are discussed to provide a basis to the understanding of the chemical evolution of disks.
\end{abstract}

Keywords. galaxies: abundances, spirals and evolution

\section{Introduction}

The study of the chemical evolution of nearby spiral galaxies is very important to improve our knowledge about the main ingredients used in chemical evolution models and test the basic assumptions made for modelling our Galaxy. M31 and M33 are other spiral members of the Local Group of galaxies and during the last years many observational studies have been made to investigate the chemical and dynamical properties of these neighbour systems. New surveys (Braun et al. 2009, Magrini et al. 2007, 2009) contributed to the analysis of different stellar populations and provided more accurate data to constrain the chemical evolution models.

The disks of M31 and M33 are similar to the Milky Way disk but some observational constraints, such as the present day gas distribution, can only be explained by assuming different star formation histories for these galaxies. The star formation rate (SFR) is one of the most important parameters regulating the chemical evolution of galaxies (Matteucci \& Recchi 2001, Boissier et al. 2003) together with the initial mass function.

Another important mechanism is the "inside-out" disk formation that is very important to reproduce the radial abundance gradients (see Colavitti et al. 2008 for the most recent paper on the subject). A faster formation of the inner disk relative to the outer disk 
was proposed by Matteucci \& François (1989) and supported in the following years by Boissier \& Prantzos (1999) and Chiappini et al. (2001).

\section{Results and Discussion}

We adopt an one-infall chemical evolution model where the Galactic disk forms insideout by means of infall of a pre-enriched gas (to take into account the effect of the halothick disk evolution), and we test different thresholds and efficiencies in the SFR. The model is scaled to the disk properties of three Local Group galaxies (the Milky Way, M31 and M33) by varying its dependence on the star formation (SF) efficiency and the time scale for the infalling gas into the disk. This model is described in details by Marcon-Uchida et al. (2009) and references therein.

Using this simple model we are able to reproduce most of the observed constraints available in the literature for the studied galaxies. The radial oxygen abundance gradients and their time evolution were studied in detail.

The most massive disks seem to have evolved faster than the less massive ones. The threshold and the efficiency in the star formation play a very important role in the chemical evolution of spiral disks and a variable efficiency along the radius can be used to regulate the star formation. The oxygen abundance gradient can steepen or flatten in time depending on the choice of this parameter.

Concerning the SF threshold effect in the radial oxygen gradients we note that it is more visible for the Milky Way and M33 than for M31. This fact is compatible with the scenario proposed by Pohlen et al. (2004) who suggest that the SF threshold can produce a truncation in the observed stellar luminosity profile of spiral discs and that low-mass galaxies should have smaller values for this radius than the massive ones.

In conclusion we find that the present day value of the oxygen abundance is more sensible to the threshold in the SFR than the efficiency in the SF and that this last parameter plays an important role in the time evolution of the gradient. The variable efficiency in the SFR is also important to reproduce the present day gas distribution in the disk of galaxies with a remarkable presence of spiral arms. A correlation between the galaxy mass and the star density profile can be seen when observing that the stellar distribution along the galactic radius gets steeper from the most massive (M31) to the lower massive one (M33).

We acknowledge finnancial support from the CNPq, CAPES and FAPESP.

\section{References}

Boissier, S. \& Prantzos, N. 1999, MNRAS, 307, 857

Boissier, S., Prantzos, N., Boselli, A., \& Gavazzi, G. 2003, MNRAS, 346, 1215

Braun, R., Thilker, D. A., Walterbos, R. A. M., \& Corbelli, E. 2009, ApJ, 695, 937

Chiappini C., Matteucci F., \& Romano D., 2001, ApJ, 554, 1044

Colavitti, E., Matteucci, F., \& Murante, G. 2008, A\&A, 483, 401

Magrini, L., Corbelli, E., \& Galli, D. 2007, $A \mathscr{E} A$, 470, 843

Magrini, L., Stanghellini, L., \& Villaver, E. 2009, ApJ, 696, 729

Marcon-Uchida, M. M., Matteucci, F. \& Costa, R. D. D. 2009, A\& $A$ submitted.

Matteucci, F. \& Francois, P. 1989, MNRAS, 239, 885

Matteucci, F. \& Recchi, S. 2001, ApJ, 558, 351

Pohlen, M., Beckman, J. E., Hüttemeister, S., Knapen, J. H., Erwin, P., \& Dettmar, R.-J. 2004, Penetrating Bars Through Masks of Cosmic Dust, 319, 713 